\title{
AN INVESTIGATION OF THE MANIPULATION OF DRUGS TO GET THE REQUIRED DOSE IN NEONATAL PRACTICE
}

\author{
R.H. Richey ${ }^{1}$, U.U. Shah ${ }^{1}$, C.E. Barker ${ }^{1}$, C. Donnell ${ }^{1}$, J.L. Ford $^{2}$, M. Peak ${ }^{1}$, A.J. Nunn ${ }^{1}$, M.A. Turner ${ }^{3}$ \\ ${ }^{1}$ Alder Hey Children's NHS Foundation Trust, ${ }^{2}$ Liverpool John Moores University, ${ }^{3}$ Liverpool Women's \\ Hospital, Liverpool, UK
}

Background and aims: The lack of commercially-available, age-appropriate formulations means that medicines need to be manipulated to achieve the required dose for neonates. To date there has been no systematic assessment of the nature of manipulations or of practice issues associated with manipulations

Methods: An observational study on one regional and two smaller neonatal units supplemented by a national survey and a systematic review.

Results: The observational study located 58 manipulations involving 18 different drugs in neonatal units; there were 44 questionnaire respondents from nurses on neonatal units who identified 34 manipulations involving 15 different drugs. None of the manipulations were supported by evidence from the systematic review. Of the 92 manipulations identified 58\% were of intravenous (IV) $20 \%$ were sachets, $6.5 \%$ tablets, $6.5 \%$ transdermal patches and $5.4 \%$ suppositories. The three IV drugs identified by both methods were vancomycin, ranitidine and midazolam; another 20 IV drugs were only identified by one of the methods. Eighteen $(43 \%)$ of the questionnaire respondents noted possible concerns with drug manipulations, these related to dose accuracy, the use of dilution, the importance of good communication with colleagues and the value of written guidance.

Conclusions: Manipulations to administer the required dose for neonates include high-risk practices which are not supported by evidence. We report the pattern of manipulations and practical issues which will need to be accounted for in the development of guidelines for neonatal units. Multiple methods were needed to describe practice: evaluating change will be complex. 